\title{
Incretin Kinetics Before and After Miglitol in Japanese Patients With Late Dumping Syndrome
}

\author{
Mari Amagai ${ }^{\mathrm{a}, \mathrm{e}}$, Hirohisa Tsuchiya ${ }^{\mathrm{a}, \mathrm{e}, \mathrm{f}}$, Yukari Chiba ${ }^{\mathrm{a}}$, Jun Suzuki ${ }^{\mathrm{b}}$, \\ Jo Nagakura $^{\mathrm{b}}$, Erina Shigematsuc ${ }^{\mathrm{c}}$, Tadashi Yamakawa ${ }^{\mathrm{b}}$, \\ Yasuo Terauchi ${ }^{\mathrm{d}}$
}

\begin{abstract}
Background: In patients with late dumping syndrome following gastrectomy, it has been reported that hypoglycemia occurs due to inhibition of glucagon secretion as a result of excessive insulin production facilitated by an increase in glucagon-like peptide-1 (GLP-1).

Methods: To determine the kinetics of incretins in Japanese patients with late dumping syndrome, an oral glucose tolerance test was carried out before and after miglitol administration, and the kinetics of insulin and incretins were analyzed.

Results: After miglitol administration, there was improvement of hypoglycemia and early phase insulin secretion, with persistent excessive insulin secretion being minimized. These findings revealed that miglitol inhibited rapid excessive influx of carbohydrates into the blood and persistent elevation of GLP-1, resulting in improvement of early phase insulin secretion and minimizing persistent excessive insulin secretion.
\end{abstract}

Conclusions: Eating frequent small meals is generally effective for late dumping syndrome, but patients often find it difficult to continue such a regimen. Based on the present analysis of incretin kinetics, miglitol may be a useful treatment option for late dumping syndrome.

Keywords: Late dumping syndrome; Glucagon-like peptide-1; Glucose-dependent insulinotropic polypeptide; $\alpha$-glucosidase inhibitor

Manuscript submitted July 17, 2017, accepted July 28, 2017

aDepartment of Endocrinology and Diabetes, Yokosuka City Hospital, Yokosuka, Japan

${ }^{b}$ Department of Endocrinology and Diabetes, Yokohama City University Medical Center, Yokohama, Japan

'Department of Diabetes and Endocrinology, National Hospital Organization Yokohama Medical Center, Yokohama, Japan

${ }^{\mathrm{d}}$ Department of Endocrinology and Metabolism, Yokohama City University School of Medicine, Yokohama, Japan

${ }^{\mathrm{e}}$ These authors contributed equally to this work.

${ }^{f}$ Corresponding Author: Hirohisa Tsuchiya, Department of Endocrinology and Diabetes, Yokosuka City Hospital, 1-3-2 Nagasaka, Yokosuka 240-0195, Japan. Email: hirotsuchiya-dm@umin.ac.jp

doi: https://doi.org/10.14740/jocmr3135w

\section{Introduction}

Due to rapid emptying of the remnant stomach following gastrectomy, patients may experience both systemic and abdominal symptoms after meals, and this is called dumping syndrome. Depending on the timing of symptom onset in relation to meals, this syndrome is classified as early or late dumping and late dumping is also known as late postprandial hypoglycemia. Late dumping syndrome is less frequent than early dumping syndrome [1], with early dumping syndrome being observed in $10-20 \%$ of patients following gastrectomy, while $5-16 \%$ of patients develop late dumping syndrome. In late dumping syndrome, symptoms occur 2 - $3 \mathrm{~h}$ after meals in association with persistent hypersecretion of insulin and consequent hypoglycemia. Late dumping syndrome is thought to be related to increased secretion of glucagon-like peptide-1 (GLP-1) as a result of rapid movement of food through the gastrointestinal tract, leading to hypersecretion of insulin promoted by GLP-1 and reactive hypoglycemia caused by inhibition of glucagon secretion [2].

Recently, there have been reports that late dumping syndrome can be improved by treatment with $\alpha$-glucosidase inhibitors ( $\alpha$-GIs) [3-5]. For example, it was reported that postprandial hypoglycemia can be prevented through suppression of insulin hypersecretion by using acarbose to reduce early phase GLP-1 secretion [6]. Unlike acarbose, miglitol is absorbed in the upper small intestine, making its effect more pronounced in the proximal small bowel and weaker in the distal small bowel [7]. Late dumping syndrome is partly due to rapid movement of food into the upper small intestine after meals, and miglitol may be effective for this syndrome because of its selective effect on the proximal small bowel. However, there is little detailed information about the effect of $\alpha$-GIs, including the influence on incretin secretion kinetics, in Japanese patients with late dumping syndrome and there have been no reports regarding miglitol.

Therefore, as a preliminary study, we tried to elucidate incretin hormone kinetics in Japanese patients with late dumping syndrome before and after administration of miglitol. We conducted a 75-g oral glucose tolerance test (OGTT) before and after dosing with miglitol in four patients with late dumping syndrome and compared the blood levels of glucose, insulin, and incretin hormones. Using continuous glucose monitoring (CGM), we also compared the changes of blood glucose before and after miglitol treatment in three patients. 
Table 1. Clinical Features and Demographic Profile

\begin{tabular}{lllll}
\hline Patients & Case 1 & Case 2 & Case 3 & Case 4 \\
\hline Sex & Male & Male & Female & Female \\
Age (years) & 73 & 75 & 71 & 83 \\
Height $(\mathrm{cm})$ & 165.7 & 159.4 & 152.8 & 155.7 \\
Body weight $(\mathrm{kg})$ & 50.5 & 49.7 & 43.1 & 39.6 \\
Body mass index $\left(\mathrm{kg} / \mathrm{m}^{2}\right)$ & 18.4 & 19.7 & 18.5 & 16.3 \\
Operation & $\begin{array}{l}\text { Total gastrectomy } \\
\text { (Rouex-en-Y) }\end{array}$ & $\begin{array}{l}\text { Total gastrectomy } \\
\text { (Roux-en-Y) }\end{array}$ & $\begin{array}{l}\text { Total gastrectomy } \\
\text { (unknown) }\end{array}$ & $\begin{array}{l}\text { Partial gastrectomy and duodenectomy } \\
\text { (unknown) }\end{array}$ \\
\hline
\end{tabular}

\section{Patients and Methods}

The subjects were four patients who were referred to our department with suspected late dumping syndrome and were admitted to Yokohama City University Medical Center between March 2012 and July 2013. An OGTT was performed and blood samples were collected at 0,30,60, 90, 120, and $180 \mathrm{~min}$. Then miglitol (50 mg) was administered and the OGTT was performed before and after administration. Using each blood sample collected, the following variables were measured: blood glucose $(\mathrm{mg} / \mathrm{dL})$, insulin $(\mu \mathrm{U} / \mathrm{mL})$ (Elecsys insulin reagent, Roche Diagnostics), total glucose-dependent insulinotropic peptide (GIP) (pg/mL) (human GIP (total) ELISA kit, Merck Millipore), active GLP-1 (pmol/mL) (glucagon like peptide-1 ELISA kit, Merck Millipore), and glucagon (pg/mL) (glucagon RIA kit, Merck Millipore). For measuring the active form of GLP-1, $20 \mu \mathrm{L}$ of a dipeptidyl peptidase- 4 (DPP-4) inhibitor (DPP-4 inhibitor, Merck Millipore, $10 \mu \mathrm{L}$ per $1.0 \mathrm{~mL}$ of blood, $1 \%$ concentration used in the blood) was added to the blood collection tube immediately before blood sampling. Immediately after mixing, the blood samples were centrifuged under refrigeration to separate plasma that was stored frozen at $-20^{\circ} \mathrm{C}$. Following solid phase extraction as pretreatment, measurements were conducted by enzymelinked immunosorbent assay (ELISA). For measurement of total GIP, samples were also collected in the blood collection tubes and centrifuged immediately under refrigeration to separate plasma that was stored frozen at $-20{ }^{\circ} \mathrm{C}$ until measurement by ELISA. Glucagon was measured by using RIA2 antibody. Furthermore, fluctuations of blood glucose before and after miglitol administration were assessed by CGM (CGMS ${ }^{\circledR}$ System Gold ${ }^{\mathrm{TM}}$, iPro2 ${ }^{\mathrm{TM}}$; Medtronic). Patients provided informed consent to these investigations, which were performed as part of routine clinical practice. Costs for measurement of incretins and glucagon were covered by a research grant.

\section{Results}

\section{Clinical features and demographic profile}

Four subjects were enrolled in this study. Their clinical features and demographic profile are shown in Table 1. Two subjects were men and two were women. Three subjects had undergone surgery for gastric cancer and one subject had received surgery for gastric ulcer. Roux-en-Y anastomosis was performed in two subjects, but the details of the surgical procedure were unknown in the other two patients. When the subjects were hospitalized, it was 6 - 20 years after surgery and they all showed a decrease in blood glucose to $40-50 \mathrm{mg} / \mathrm{dL}$ at 2 - $3 \mathrm{~h}$ after meals associated with symptoms of hypoglycemia. Because these symptoms were suspected to be caused by late dumping syndrome, an OGTT was performed to confirm the diagnosis.

\section{Blood glucose and insulin levels in the OGTT before and after miglitol}

In case 3 , in the OGTT before administration of miglitol, the blood glucose level was elevated to $279 \mathrm{mg} / \mathrm{dL}$ at $30 \mathrm{~min}$, followed by a marked decrease to $46 \mathrm{mg} / \mathrm{dL}$ at $120 \mathrm{~min}$. In the OGTT following administration of miglitol, the blood glucose level was decreased to $168 \mathrm{mg} / \mathrm{dL}$ at $30 \mathrm{~min}$. At $180 \mathrm{~min}$, the blood glucose level was improved to $80 \mathrm{mg} / \mathrm{dL}$. Similarly, in case 4 , in the OGTT before administration of miglitol, the blood glucose level was elevated to $222 \mathrm{mg} / \mathrm{dL}$ at $60 \mathrm{~min}$, followed by a marked decrease to $27 \mathrm{mg} / \mathrm{dL}$ at $180 \mathrm{~min}$. In the OGTT following administration of miglitol, the blood glucose level was decreased to $185 \mathrm{mg} / \mathrm{dL}$ at $60 \mathrm{~min}$. At $180 \mathrm{~min}$, the blood glucose level was improved to $55 \mathrm{mg} / \mathrm{dL}$. In case 1, blood glucose level decreased $30 \mathrm{~min}$ after administration, and blood glucose level after 180 min improved. However, in case 2 , there was no obvious difference in blood glucose level before and after administration (Table 2).

In cases 1, 3 and 4, before miglitol administration, the peak insulin level was observed at $60 \mathrm{~min}$, while the peak was at $30 \mathrm{~min}$ following miglitol administration. In addition, the insulinogenic index increased from 0.37 before miglitol administration to 1.64 after administration in case 1 , indicating improvement of early phase insulin secretion. Similarly, in cases 2 and 3, the insulinogenic index improved.

Total GIP, active GLP-1, and glucagon in the OGTT before and after miglitol

In the OGTT before miglitol administration, the peak level of active GLP-1 was observed at $30 \mathrm{~min}$. In cases 3 and 4, the 
Table 2. Blood Glucose and Insulin Levels in the OGTT Before and After Miglitol

\begin{tabular}{|c|c|c|c|c|c|}
\hline & 0 min & $30 \mathrm{~min}$ & $60 \mathrm{~min}$ & $120 \mathrm{~min}$ & $180 \mathrm{~min}$ \\
\hline \multicolumn{6}{|c|}{ Blood glucose (mg/dL) } \\
\hline \multicolumn{6}{|c|}{ OGTT before miglitol } \\
\hline Case 1 & 104 & 276 & 278 & 154 & 65 \\
\hline Case 2 & 88 & 240 & 179 & 116 & 70 \\
\hline Case 3 & 89 & 279 & 175 & 46 & 69 \\
\hline Case 4 & 81 & 171 & 222 & 114 & 27 \\
\hline \multicolumn{6}{|c|}{ OGTT after miglitol } \\
\hline Case 1 & 102 & 229 & 159 & 87 & 87 \\
\hline Case 2 & 88 & 191 & 200 & 89 & 57 \\
\hline Case 3 & 87 & 168 & 58 & 70 & 80 \\
\hline Case 4 & 77 & 147 & 185 & 70 & 55 \\
\hline \multicolumn{6}{|c|}{ Insulin $(\mu \mathrm{U} / \mathrm{mL})$} \\
\hline \multicolumn{6}{|c|}{ OGTT before miglitol } \\
\hline Case 1 & 4.1 & 66.9 & 287.7 & 146.5 & 13 \\
\hline Case 2 & 3.6 & 99.18 & 84.7 & 21.5 & 2.9 \\
\hline Case 3 & 4.3 & 88.5 & 195.9 & 11 & 8.6 \\
\hline Case 4 & 0.8 & 91.4 & 119.8 & 34.3 & 3 \\
\hline \multicolumn{6}{|c|}{ OGTT after miglitol } \\
\hline Case 1 & 3.1 & 211.2 & 70.5 & 18.6 & 5.4 \\
\hline Case 2 & 4.7 & 238.2 & 377.5 & 36.8 & 6.3 \\
\hline Case 3 & 1.6 & 64.1 & 8.4 & 2.1 & 2 \\
\hline Case 4 & 0.5 & 63.1 & 30.7 & 3.9 & 1.7 \\
\hline \multicolumn{6}{|c|}{ Insulinogenic index } \\
\hline \multicolumn{6}{|c|}{ OGTT before miglitol } \\
\hline Case 1 & 0.37 & & & & \\
\hline Case 2 & 0.63 & & & & \\
\hline Case 3 & 0.44 & & & & \\
\hline Case 4 & 1.01 & & & & \\
\hline \multicolumn{6}{|c|}{ OGTT after miglitol } \\
\hline Case 1 & 1.64 & & & & \\
\hline Case 2 & 2.27 & & & & \\
\hline Case 3 & 0.77 & & & & \\
\hline Case 4 & 0.89 & & & & \\
\hline
\end{tabular}

GLP-1 level at $30 \mathrm{~min}$ decreased from 50.6 and $54.9 \mathrm{pmol} / \mathrm{mL}$ before miglitol administration to 19.5 and $12.9 \mathrm{pmol} / \mathrm{mL}$ after administration, respectively. However, no obvious change was observed in cases 1 and 2 (Table 3).

The peak total GIP level was observed at 30 min during the OGTT performed before miglitol administration. In all cases, in the OGTT after administration of miglitol, the GIP level was significantly decreased at $30 \mathrm{~min}$.

In both OGTTs, there was a paradoxical increase in glucagon after glucose loading and this increase persisted up to 180 min. Glucagon values tended to be lower after administration of miglitol.

\section{Results of CGM before and after miglitol}

The 24-h blood glucose levels before and after miglitol administration were assessed in three patients by CGM. Before miglitol administration, the maximum blood glucose levels were 374,271 and $220 \mathrm{mg} / \mathrm{dL}$, respectively. After miglitol administration, the maximum blood glucose levels were 142, 231 and $116 \mathrm{mg} / \mathrm{dL}$, respectively. Before miglitol administration, 
Table 3. Total GIP, Active GLP-1 and Glucagon in the OGTT Before and After Miglitol

\begin{tabular}{|c|c|c|c|c|c|}
\hline & 0 min & $30 \mathrm{~min}$ & $60 \mathrm{~min}$ & $120 \mathrm{~min}$ & $180 \mathrm{~min}$ \\
\hline \multicolumn{6}{|c|}{ Active GLP-1 (pmol/mL) } \\
\hline \multicolumn{6}{|c|}{ OGTT before miglitol } \\
\hline Case 1 & 2 & 38.7 & 19.6 & 10.9 & 2.6 \\
\hline Case 2 & 3.8 & 88.9 & 36 & 16.1 & 3.8 \\
\hline Case 3 & 2 & 50.6 & 15.2 & 14.2 & 3.7 \\
\hline Case 4 & 2 & 54.9 & 39.2 & 7.2 & 2 \\
\hline \multicolumn{6}{|c|}{ OGTT after miglitol } \\
\hline Case 1 & 2 & 42.4 & 7.8 & 16.7 & 3.8 \\
\hline Case 2 & 2 & 98.8 & 18.7 & 7 & 4.6 \\
\hline Case 3 & 2 & 19.5 & 4.1 & 3.3 & 2.7 \\
\hline Case 4 & 2 & 12.9 & 5 & 3 & 3.1 \\
\hline \multicolumn{6}{|l|}{ Total GIP (pg/mL) } \\
\hline \multicolumn{6}{|c|}{ OGTT before miglitol } \\
\hline Case 1 & 48 & 357 & 213 & 82.5 & 69.2 \\
\hline Case 2 & 64.7 & 598 & 214 & 134 & 81.5 \\
\hline Case 3 & 58.5 & 520 & 210 & 123 & 81.5 \\
\hline Case 4 & 41.8 & 530 & 437 & 189 & 71 \\
\hline \multicolumn{6}{|c|}{ OGTT after miglitol } \\
\hline Case 1 & 52.2 & 278 & 132 & 71.9 & 64.6 \\
\hline Case 2 & 59.5 & 476 & 255 & 125 & 79.6 \\
\hline Case 3 & 66.9 & 277 & 151 & 87.3 & 63.5 \\
\hline Case 4 & 33.7 & 262 & 213 & 53.7 & 32.3 \\
\hline \multicolumn{6}{|l|}{ Glucagon (pg/mL) } \\
\hline \multicolumn{6}{|c|}{ OGTT before miglitol } \\
\hline Case 1 & 63 & 151 & 127 & 106 & 122 \\
\hline Case 2 & 78 & 130 & 136 & 140 & 147 \\
\hline Case 3 & 66 & 133 & 97 & 97 & 107 \\
\hline Case 4 & 75 & 92 & 103 & 96 & 90 \\
\hline \multicolumn{6}{|c|}{ OGTT after miglitol } \\
\hline Case 1 & 54 & 126 & 115 & 108 & 111 \\
\hline Case 2 & 101 & 150 & 156 & 130 & 150 \\
\hline Case 3 & 54 & 69 & 67 & 75 & 64 \\
\hline Case 4 & 57 & 82 & 72 & 64 & 65 \\
\hline
\end{tabular}

the minimum blood glucose levels were 62,40 and $59 \mathrm{mg} / \mathrm{dL}$, respectively. After miglitol administration, the minimum blood glucose levels were 65, 68 and $66 \mathrm{mg} / \mathrm{dL}$, respectively. From these results, both the maximum and minimum glucose levels were improved by miglitol. Figure 1 shows a typical example of the changes in blood glucose by CGM. After administration of miglitol, there was improvement of blood glucose as well as the symptoms of hypoglycemia (Table 4).

\section{Discussion}

In this study, miglitol was administered to patients with late dumping syndrome as a complication of gastrectomy. We performed the OGTT before and after administration of miglitol, and we evaluated blood glucose, insulin, and incretin hormones, and glucagon kinetics. Following miglitol administration, hypoglycemia was abolished and hypersecretion of insulin and GLP-1 was inhibited.

Miglitol is an $\alpha-G 1$ and these drugs improve postprandial hyperglycemia by slowing the digestion of disaccharides to monosaccharides, thereby shifting the site of glucose absorption from the upper small intestine to the middle/lower small intestine and thus delaying absorption. Acarbose and voglibose are largely unabsorbed and act throughout the entire small intestine. Because miglitol is absorbed in the lower small in- 


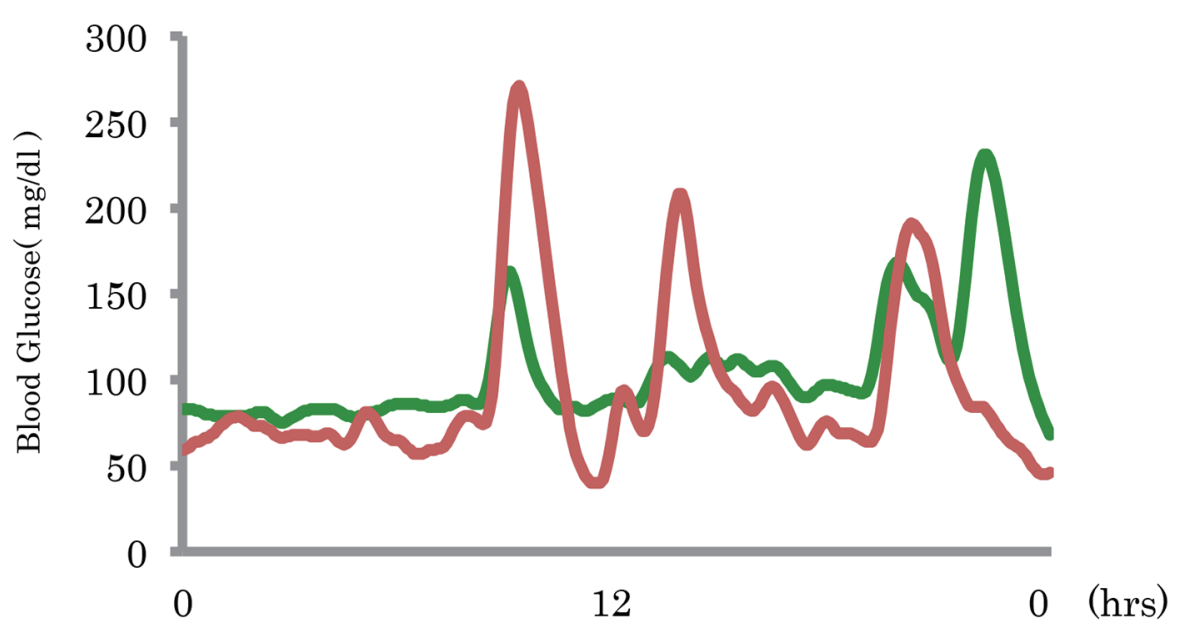

Figure 1. A typical example of the changes in blood glucose before and after miglitol administration.

testine, its concentration is higher in the upper small intestine and its effect is attenuated in the distal small bowel [7]. Accordingly, miglitol causes less gastrointestinal symptoms than other $\alpha-G 1 s$ and higher doses can be administered, thus markedly reducing early phase postprandial glycemic excursions. In patients with type 2 diabetes who have a normal gastrointestinal tract, it has been shown that postprandial inhibition of GIP is greater with miglitol than voglibose and is linked to an increase in GLP-1 secretion at $1-2 \mathrm{~h}$ after meals [8]. In patients with late dumping syndrome, miglitol may also be more effective than other drugs for inhibiting rapid absorption of carbohydrates and preventing hypoglycemia over time. However, the present study did not compare miglitol with other medications, and further studies will be needed to investigate this hypothesis. In general, reducing the size of meals and eating more frequently throughout the day are effective countermeasures for late dumping syndrome, but patients need to eat 5 - 6 meals a day and it is often difficult for them to continue such a regimen. For patients struggling to comply with eating frequent meals, administration of an $\alpha$-GI could be useful. The present study showed improvement of blood glucose following miglitol administration. In particular, there was a decrease

Table 4. Results of CGM Before and After Miglitol

\begin{tabular}{|llll}
\hline Patients & Case 1 & Case 2 & Case 3 \\
\hline Before & & & \\
Mean (mg/dL) & 153 & 94 & 97 \\
Maximum (mg/dL) & 374 & 271 & 220 \\
Minimum (mg/dL) & 62 & 40 & 59 \\
SD (mg/dL) & 73 & 49 & 28 \\
After & & & \\
Mean (mg/dL) & 98 & 105 & 81 \\
Maximum (mg/dL) & 142 & 231 & 116 \\
Minimum (mg/dL) & 65 & 68 & 66 \\
SD (mg/dL) & 20 & 33 & 10 \\
\hline
\end{tabular}

at $30 \mathrm{~min}$. Regarding insulin secretion, the peak insulin level shifted from 60 to $30 \mathrm{~min}$. Therefore, miglitol facilitated early phase insulin secretion, resulting in the peak insulin level shifting to $30 \mathrm{~min}$ and improving persistent insulin hypersecretion. In turn, this led to a significant decrease in the blood glucose from 0 to $30 \mathrm{~min}$. It has been reported that the total GLP-1 concentration increases to $3-6 \mathrm{pmol} / \mathrm{mL}$ after the OGTT in healthy Japanese subjects [9]. In patients with late dumping syndrome, including those examined in this study, hypersecretion of GLP-1 was suspected and a significant decrease in GLP-1 secretion occurred following miglitol administration. GLP-1 secretion is thought to have early and late phases, with the early phase being linked to release of humoral factors from upper gastrointestinal tract and from autonomic nervous system. It has been reported that GLP-1-secreting L cells exist in the upper gastrointestinal tract and respond to direct stimulation by nutrients [10]. Therefore, the overall inhibition of GLP-1 hypersecretion observed in this study was probably brought about by administration of miglitol, which inhibited absorption of carbohydrates in the upper gastrointestinal tract, thereby inhibiting GLP-1 secretion in the early phase. Likewise, in the evaluation using CGM, miglitol administration resulted in improvement of maximum blood glucose level and minimum blood glucose level. These results are in agreement with data reported previously, indicating that miglitol inhibits excessive secretion of active GLP-1 and thus prevents insulin hypersecretion and the onset of hypoglycemia.

With regard to GIP, there are conflicting reports that its level does not change [11] or that it increases [12] in patients with late dumping syndrome. While there is not yet a consensus about this issue, one study investigated the response of GIP to meals in Japanese patients who underwent gastrectomy with different reconstruction methods [13]. This study showed that GIP secretion increased in the following order: healthy subjects, patients with Billroth I reconstruction following gastrectomy, patients with Billroth II reconstruction following gastrectomy, and patients with total gastrectomy. Because changes in the passage of food through the upper gastrointestinal tract have a marked impact on GIP secretion, it is predicted 
that patients with late dumping syndrome would also show an increased secretory response of GIP. In support, the present study also revealed excessive secretion of GIP at $30 \mathrm{~min}$ during the OGTT performed before miglitol administration. Following miglitol administration, there was a significant decrease of GIP at $30 \mathrm{~min}$, indicating that miglitol inhibited GIP secretion. Regarding the effects of the different reconstruction methods, the activity of miglitol may be slightly weaker in patients who have undergone surgery with Billroth II reconstruction or Roux-en-Y anastomosis, both of which result in no passage of the drug through the duodenum, as compared to those who have undergone surgery with Billroth I reconstruction. However, it is predicted that the Billroth I method results in greater secretion of GIP compared with the other methods. Thus, it is possible that the differences in the effect of miglitol are related to the indirect impact on GLP-1 secretion of factors associated with each reconstruction method. Although analysis of the influence of each reconstruction method could not be carried out in the present study due to the small number of patients who had undergone surgery with either Roux-en-Y anastomosis or an unknown procedure, these analyses need to be performed in the future. It has been reported that hypersecretion of GLP-1 occurs in patients with late dumping syndrome [2] leading to the inhibition of glucagon secretion in response to hypoglycemia and hyperinsulinemia, and contributing to induction of hypoglycemia. However, another study [14] found hypersecretion of glucagon in patients. Similarly, hypersecretion of glucagon was noted following the OGTT in the present study. Although high glucagon levels persisted after miglitol administration, there was a tendency for glucagon to decrease compared with before administration. Known effects of GLP1 on the digestive tract include inhibition of gastric emptying, inhibition of gastric acid secretion, and inhibition of pancreatic exocrine secretion. It has been shown that these effects on the digestive organs are dependent on vagal activity [15], and the effects of GLP-1 are impaired in patients who have undergone total vagotomy with pyloroplasty [16]. A previous report [2] also suggested that hypoglycemia is partly induced by inhibition of glucagon secretion. However, another study indicated that GIP acts on $\alpha$ cells of the pancreas and enhances glucagon secretion [17]. The subjects of this study are likely to have undergone total vagotomy during their operative procedures. Because vagotomy impairs GLP-1 activity, hypersecretion of glucagon in these subjects is likely to have resulted from the effects of GIP. Glucagon secretion tended to decrease following miglitol administration, probably due to inhibition of GIP secretion. However, the present study could not determine the kinetics of GIP and glucagon during hypoglycemia in patients with late dumping syndrome, and further investigation is needed in the future.

Recently, metabolic surgery has attracted attention with an increase in obesity [18]. The obesity rates in Japan and the Unites States are 3.5\% and 35.9\%, respectively. Although the rate is lower in Japan compared to the United States, the mean BMI of Japanese males is clearly on the rise in every age group. Therefore, it is likely that the future demand for weight loss surgery will increase in Japan as well. An increase in GLP-1 during the OGTT has been reported following laparoscopic sleeve gastrectomy [19], while hypersecretion of in- cretins and insulin with the onset of postprandial hypoglycemia were noted following Roux-en-Y gastric bypass surgery $[20,21]$. The possibility cannot be ruled out that concomitant postoperative dumping syndrome was present in the reported patients via a mechanism similar to that in the present study. In the future, weight loss surgery may be increasingly carried out in Japan and this may increase the number of patients with late dumping syndrome. Because the actions of miglitol are different from those of other $\alpha$-GIs, it is hoped that miglitol can prevent persistent insulin secretion in these patients by inhibiting rapid influx of carbohydrates and an excessive persistent increase in GLP-1, thus facilitating early insulin secretion following its administration. However, there have been reports of intestinal obstruction in patients treated with acarbose [22] and voglibose [23]. Because there was also a similar case report for miglitol [5], close attention should be paid to the risk of intestinal obstruction when administering $\alpha$-GIs following abdominal surgery.

In patients with late dumping syndrome, we found that administration of the $\alpha$-GI miglitol is likely to improve hypoglycemia. However, the present study was based on a small number of patients and only short-term effects were monitored. This study was also limited by the lack of comparison with other $\alpha$-GIs. Therefore, further comparative studies on a larger scale are needed in the future.

\section{Conclusion}

We analyzed the kinetics of incretin secretion in patients with late dumping syndrome who underwent treatment with miglitol and showed improvement of hypoglycemia. This study revealed that hypersecretion of GLP-1 was inhibited by administration of miglitol, reducing persistent excessive secretion of insulin and thus improving hypoglycemia. However, the roles of GIP and glucagon are still unclear, and more studies are needed to elucidate these points in the future.

\section{Acknowledgments}

We thank Dr. Yamakawa and all the physicians who participated in this study.

\section{Conflicts of Interest}

No other potential conflicts of interest relevant to this article were reported.

\section{References}

1. Hideaki K. Dumping syndrome and afferent loop syndrome. Surgical Therapy. 2011;104:756-761.

2. Miholic J, Orskov C, Holst JJ, Kotzerke J, Meyer HJ. Emptying of the gastric substitute, glucagon-like peptide-1 (GLP-1), and reactive hypoglycemia after total 
gastrectomy. Dig Dis Sci. 1991;36(10):1361-1370.

3. Hasegawa T, Yoneda M, Nakamura K, Ohnishi K, Harada H, Kyouda T, Yoshida Y, et al. Long-term effect of alphaglucosidase inhibitor on late dumping syndrome. J Gastroenterol Hepatol. 1998;13(12):1201-1206.

4. Kondo Y, Toyoda K, Santo T, Fujii J, Fukushima M, Inagaki N, Yasuda K. A patient who developed symptomatic reactive hypoglycemia 14 years after total gastrectomy and was successfully treated with miglitol. Diabetol Int. 2012;4:66-70.

5. Fujita Y, Tamada D, Kozawa J, Kobayashi Y, Sasaki S, Kitamura T, Yasuda T, et al. Successful treatment of reactive hypoglycemia secondary to late dumping syndrome using miglitol. Intern Med. 2012;51(18):2581-2585.

6. Valderas JP, Ahuad J, Rubio L, Escalona M, Pollak F, Maiz A. Acarbose improves hypoglycaemia following gastric bypass surgery without increasing glucagon-like peptide 1 levels. Obes Surg. 2012;22(4):582-586.

7. Scott LJ, Spencer CM. Miglitol: a review of its therapeutic potential in type 2 diabetes mellitus. Drugs. 2000;59(3):521-549.

8. Narita T, Yokoyama H, Yamashita R, Sato T, Hosoba M, Morii T, Fujita H, et al. Comparisons of the effects of 12-week administration of miglitol and voglibose on the responses of plasma incretins after a mixed meal in Japanese type 2 diabetic patients. Diabetes Obes Metab. 2012;14(3):283-287.

9. Yamane S, Harada N, Hamasaki A, Muraoka A, Joo E, Suzuki K, Nasteska D, et al. Effects of glucose and meal ingestion on incretin secretion in Japanese subjects with normal glucose tolerance. J Diabetes Investig. 2012;3(1):80-85.

10. Schirra J, Katschinski M, Weidmann C, Schafer T, Wank U, Arnold R, Goke B. Gastric emptying and release of incretin hormones after glucose ingestion in humans. J Clin Invest. 1996;97(1):92-103.

11. Lawaetz O, Blackburn AM, Bloom SR, Aritas Y, Ralphs DN. Gut hormone profile and gastric emptying in the dumping syndrome. A hypothesis concerning the pathogenesis. Scand J Gastroenterol. 1983;18(1):73-80.

12. Schattenmann G, Ebert R, Siewert R, Creutzfeldt W. Different response of gastric inhibitory polypeptide to glucose and fat from duodenum and jejunum. Scand J Gastroenterol. 1984;19(2):260-266.

13. Takemura J, Seino Y, Yamamura T, Tsuda K, Seino S, Itoh
$\mathrm{N}$, Imura $\mathrm{H}$. The role of endogenous gastric inhibitory polypeptide in the enteroinsular axis. J Clin Endocrinol Metab. 1982;54(5):909-913.

14. Rehfeld JF, Heding LG, Holst JJ. Increased gut glucagon release as pathogenetic factor in reactive hypoglycaemia? Lancet. 1973;1(7795):116-118.

15. Plamboeck A, Veedfald S, Deacon CF, Hartmann B, Wettergren A, Svendsen LB, Meisner S, et al. The effect of exogenous GLP-1 on food intake is lost in male truncally vagotomized subjects with pyloroplasty. Am J Physiol Gastrointest Liver Physiol. 2013;304(12):G1117-1127.

16. Wettergren A, Wojdemann M, Holst JJ. Glucagon-like peptide-1 inhibits gastropancreatic function by inhibiting central parasympathetic outflow. Am J Physiol. 1998;275(5 Pt 1):G984-992.

17. Meier JJ, Gallwitz B, Siepmann N, Holst JJ, Deacon CF, Schmidt WE, Nauck MA. Gastric inhibitory polypeptide (GIP) dose-dependently stimulates glucagon secretion in healthy human subjects at euglycaemia. Diabetologia. 2003;46(6):798-801.

18. Buchwald H, Oien DM. Metabolic/bariatric surgery worldwide 2011. Obes Surg. 2013;23(4):427-436.

19. Ugi S, Yamamoto H, Kusunoki C, Kamo A, Ikeda K, Hashimoto T, Nishio Y, et al. Laparoscopic sleeve gastrectomy leads to rapid improvement of glucose tolerance and insulin secretion with enhanced glucagon-like peptide(GLP-1)secretion. Diabetol Int. 2010;1:99-103.

20. Goldfine AB, Mun EC, Devine E, Bernier R, Baz-Hecht M, Jones DB, Schneider BE, et al. Patients with neuroglycopenia after gastric bypass surgery have exaggerated incretin and insulin secretory responses to a mixed meal. J Clin Endocrinol Metab. 2007;92(12):4678-4685.

21. Service GJ, Thompson GB, Service FJ, Andrews JC, Collazo-Clavell ML, Lloyd RV. Hyperinsulinemic hypoglycemia with nesidioblastosis after gastric-bypass surgery. N Engl J Med. 2005;353(3):249-254.

22. Oba K, Kudo R, Yano M, Watanabe K, Ajiro Y, Okazaki $\mathrm{K}$, Suzuki T, et al. Ileus after administration of cold remedy in an elderly diabetic patient treated with acarbose. J Nippon Med Sch. 2001;68(1):61-64.

23. Hayaishi R, Wada M, Imano E, Kanda T. [Occurrence of ileus after voglibose treatment in an elderly diabetic patient with gait disturbance caused by cerebral hemorrhage]. Nihon Ronen Igakkai Zasshi. 1996;33(8):607612. 